International Journal of Applied Mathematical Research, 1 (1) (2012) 65-76

\title{
On the Numerical Solution of Hammerstein Integral Equations using Legendre Approximation
}

\author{
N. H. Sweilam, M. M. Khader, and W. Y. Kota \\ Department of Mathematics, Faculty of Science, Cairo University, Giza, Egypt \\ Email: n_sweilam@yahoo.com \\ Department of Mathematics, Faculty of Science, Benha University, Benha, Egypt \\ Email: mohamed.khader@fsc.bu.edu.eg \\ Department of Mathematics, Faculty of Science, Mansoura University, Damietta, Egypt \\ Email: wafaa_kota@yahoo.com
}

\begin{abstract}
In this study, Legendre collocation method is presented to solve numerically the Fredholm-Hammerstein integral equations. This method is based on replacement of the unknown function by truncated series of well known Legendre expansion of functions. The proposed method converts the equation to matrix equation, by means of collocation points on the interval $[-1,1]$ which corresponding to system of algebraic equations with Legendre coefficients. Thus, by solving the matrix equation, Legendre coefficients are obtained. Some numerical examples are included to demonstrate the validity and applicability of the proposed technique.
\end{abstract}

Keywords: Fredholm-Hammerstein integral equations, Integral equation, Legendre collocation matrix method, Legendre polynomials, Volterra integral equation.

\section{Introduction}

In recent years, there has been a growing interest in the Fredholm and Volterra integral equations. This is an important branch of modern mathematics and arise frequently in many applied areas which include engineering, mechanics, physics, chemistry, astronomy, biology [1]-[5]. There are several methods for 
approximating the solution of linear and non-linear integral equations [10]-[14]. We consider the Hammerstein integral equations in the forms

$$
x(t)=f(t)+\lambda_{1} \int_{0}^{1} K_{1}(t, s) F(x(s)) d s+\lambda_{2} \int_{0}^{t} K_{2}(t, s) G(x(s)) d s,
$$

where $f(t), K_{1}(t, s)$ and $K_{2}(t, s)$ are given functions, $0 \leq t, s \leq 1$, and $\lambda_{1}, \lambda_{2}$ are arbitrary constants.

Orthogonal polynomials are widely used in applications in mathematics, mathematical physics, engineering and computer science. One of the most common set of the Legendre polynomials $P_{0}(t), P_{1}(t), \ldots, P_{N}(t)$ which are orthogonal on $[-1,1]$ with respect to the weight function $w(t)=1$. The Legendre polynomials $P_{n}(t)$ satisfy the Legendre differential equation

$$
\left(1-t^{2}\right) u^{\prime \prime}(t)-2 t u^{\prime}(t)+n(n+1) u(t)=0, \quad-1<t<1, \quad n \geq 0,
$$

and are given by the following relation

$$
P_{n}(t)=\frac{1}{2^{n}} \sum_{k=0}^{[n / 2]}(-1)^{k} \frac{(2 n-2 k) !}{(n-k) !(n-2 k) k !} t^{n-2 k}, \quad n=0,1,2, \ldots
$$

Also, the recurrence formula associated with Legendre polynomials is given with the relations,

$P_{0}(t)=1, P_{1}(t)=\frac{1}{2}\left(3 t^{2}-1\right),(n+1) P_{n+1}(t)=(2 n+1) t P_{n}(t)-n P_{n-1}(t), n \geq 1$.

Legendre polynomials occur in the solution of Laplace equation of the potential, $\nabla^{2} \Phi(x)=0$, in a charge-free region of space, using the method of separation of variables, where the boundary conditions have axial symmetry, the solution for the potential will be

$$
\Phi(r, \theta)=\sum_{l=0}^{\infty}\left[A_{l} r^{l}+B_{l} r^{-(l+1)}\right] P_{l}(\cos \theta)
$$

$A_{l}$ and $B_{l}$ are to be determined according to the boundary condition of each problem. They also appear when solving Schrödinger equation in three dimensions for a central force.

The Legendre and Chebyshev polynomials are mostly used to solve several problems of differential equations or integral equations, for example, the Legendre pesudo-spectral method is used to solve the delay and the diffusion differential equations ([6], [7]). Chebyshev polynomials are used to introduce an efficient modification of homotopy perturbation method [8]. Also, the polynomial approximation is used to solve high-order linear Fredholm integrodifferential equations with constant coefficient [11] and others ([9], [15]-[17]). 


\section{Procedure Solution using the Proposed Numerical Method}

We consider the Fredholm-Volterra integral equation (1). The function $x(t)$ may be expanded by infinite series of Legendre polynomials as follows

$$
x(t)=\sum_{n=0}^{\infty} c_{n} P_{n}(t)
$$

where $c_{n}=\left(x(t), P_{n}(t)\right)$. If we consider truncated series in Eq.(4), we obtain

$$
x(t) \simeq \sum_{n=0}^{N} c_{n} P_{n}(t)=C^{T} P(t),
$$

such that $C$ and $P$ are matrices given by

$$
C=\left[\begin{array}{llll}
c_{0} & c_{1} & \ldots & c_{N}
\end{array}\right], \quad P(t)=\left[\begin{array}{llll}
P_{0}(t) & P_{1}(t) & \ldots & P_{N}(t)
\end{array}\right]^{T} .
$$

Then we substitute the approximation Eq.(5) into Eq.(1) we get

$$
C^{T} P(t)=f(t)+\lambda_{1} \int_{0}^{1} K_{1}(t, s) F\left(C^{T} P(s)\right) d s+\lambda_{2} \int_{0}^{t} K_{2}(t, s) G\left(C^{T} P(s)\right) d s .
$$

Now, to use the Legendre collocation method which is a matrix method based on the Legendre collocation points depended by

$$
t_{i}=-1+\frac{2}{N} i, \quad i=0,1, \ldots, N
$$

we collocate Eq.(7) with the points (8) to obtain

$$
C^{T} P\left(t_{j}\right)=f\left(t_{j}\right)+\lambda_{1} \int_{0}^{1} K_{1}\left(t_{j}, s\right) F\left(C^{T} P(s)\right) d s+\lambda_{2} \int_{0}^{t_{j}} K_{2}\left(t_{j}, s\right) G\left(C^{T} P(s)\right) d s .
$$

The integral terms in Eq.(9) can be found using composite trapezoidal integration technique as:

$$
\int_{0}^{1} K_{1}\left(t_{j}, s\right) F\left(C^{T} P(s)\right) d s \cong \frac{h}{2}\left(\Omega_{1}\left(s_{0}\right)+\Omega_{1}\left(s_{m}\right)+2 \sum_{k=1}^{m-1} \Omega_{1}\left(s_{k}\right)\right),
$$

where $\Omega_{1}(s)=K_{1}\left(t_{j}, s\right) F\left(C^{T} P(s)\right), h=\frac{1}{m}$, for an arbitrary integer $m, s_{i}=$ $i h, \quad i=0,1, \ldots, m$ and

$$
\int_{0}^{t_{j}} K_{2}\left(t_{j}, s\right) G\left(C^{T} P(s)\right) d s \cong \frac{h_{j}}{2}\left(\Omega_{2}\left(\bar{s}_{0}\right)+\Omega_{2}\left(\bar{s}_{m}\right)+2 \sum_{k=1}^{m-1} \Omega_{2}\left(\bar{s}_{k}\right)\right),
$$


where $\Omega_{2}(s)=K_{2}\left(t_{j}, s\right) G\left(C^{T} P(s)\right), h_{j}=\frac{t_{j}}{m}$, for an arbitrary integer $m, \bar{s}_{i}=$ $i h$.

Eq.(9) gives $(N+1)$ system of linear or non-linear algebraic equations, which can be solved for $c_{k}, k=0,1, \ldots, N$. So the unknown function $x(t)$ can be found.

\section{Numerical Implementation}

In this section, to achive the validity, the accuracy and support our theoretical discussion of the proposed method, we give some computational results of numerical examples.

\section{Example 1.}

Consider Eq.(1) with the following functions and coefficients

$$
\begin{gathered}
f(t)=t^{3}-(6-2 e) e^{t}, \quad \lambda_{1}=1, \quad \lambda_{2}=1, \\
K_{1}(t, s)=e^{(t+s)}, \quad K_{2}(t, s)=0, \quad F(x(s))=x(s), G(x(s))=0 .
\end{gathered}
$$

Eq.(1) takes the form

$$
x(t)=t^{3}-(6-2 e) e^{t}+\int_{0}^{1} e^{(s+t)} x(s) d s .
$$

We apply the suggested method with $N=4$, and approximate the solution $x(t)$ as follows

$$
x_{N}(t)=\sum_{i=0}^{4} c_{i} P_{i}(t)=C^{T} P(t) .
$$

By the same procedure in the previous section and using Eq.(9) we have

$$
\sum_{i=0}^{4} c_{i} P_{i}\left(t_{j}\right)-\left(t_{j}^{3}-(6-2 e) e^{t_{j}}\right)-\frac{h}{2}\left(\Omega\left(s_{0}\right)+\Omega\left(s_{m}\right)+2 \sum_{k=1}^{m-1} \Omega\left(s_{k}\right)\right)=0, j=0,1,2,3,4,
$$

where $\Omega(s)=e^{\left(s+t_{j}\right)} \sum_{i=0}^{4} c_{i} P_{i}(s)$ and the nodes $s_{l+1}=s_{l}+h, l=0,1, \ldots, m$, $s_{0}=0$ and $h=\frac{1}{m}$.

Eq.(14) represents linear system of $N+1$ algebraic equations in the coefficients $c_{i}$, by solving it using the conjugate gradient method, we obtain

$$
c_{0}=-0.0048, \quad c_{1}=0.5955, \quad c_{2}=-0.0015, \quad c_{3}=0.3998, \quad c_{4}=-0.0001 .
$$

Therefore, the approximate solution of this example using Eq.(13) is given by $x(t)=-0.0048 P_{0}(t)+0.5955 P_{1}(t)-0.0015 P_{2}(t)+0.3998 P_{3}(t)-0.0001 P_{4}(t)$. 
The exact solution of this example is $x(t)=t^{3}$.

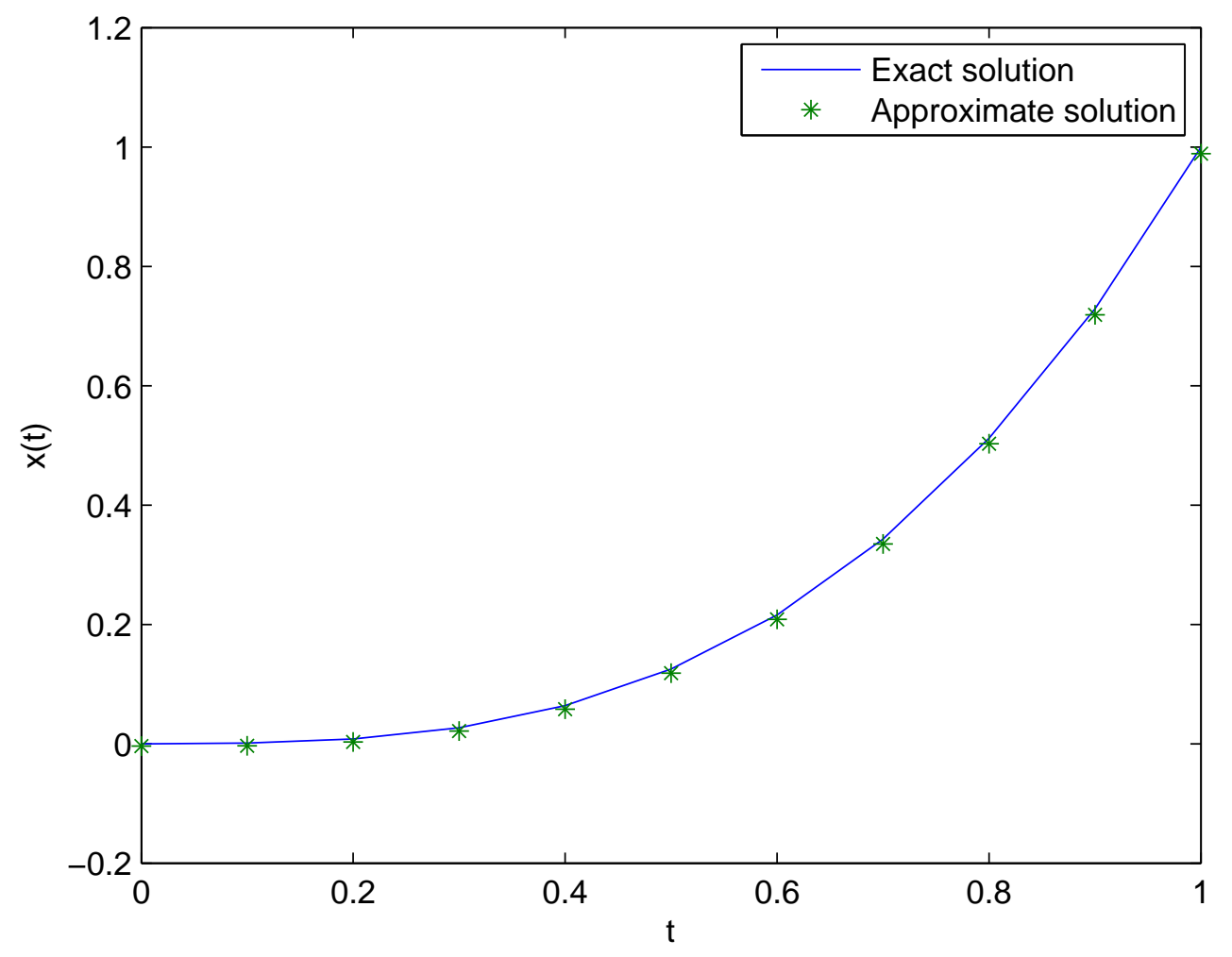

Fig.1. The behavior of the exact solution and the approximate solution at $N=4$.

The behavior of the approximate solution using the proposed method with $N=4$ and the exact solution are presented in Fig.1. From this Fig., it is clear that the proposed method can be considered as an efficient method to solve the linear integral equations.

\section{Example 2.}

Consider Eq.(1) with the following functions and coefficients

$$
\begin{gathered}
f(t)=2 t e^{t}-e^{t}+1, \quad \lambda_{1}=1, \quad \lambda_{2}=-1, \quad K_{1}(t, s)=0, \\
K_{2}(t, s)=(s+t), \quad F(x(s))=0, \quad G(x(s))=e^{x(s)} .
\end{gathered}
$$

Eq.(1) takes the following form

$$
x(t)=2 t e^{t}-e^{t}+1-\int_{0}^{t}(s+t) e^{x(s)} d s .
$$


We apply the suggested method with $N=4$, and approximate the solution $x(t)$ as follows

$$
x_{N}(t)=\sum_{i=0}^{4} c_{i} P_{i}(t)=C^{T} P(t)
$$

By the same procedure in the previous section and using Eq.(9) we have

$$
\sum_{i=0}^{4} c_{i} P_{i}\left(t_{j}\right)-f\left(t_{j}\right)+\frac{h_{j}}{2}\left(\Omega\left(s_{0}\right)+\Omega\left(s_{m}\right)+2 \sum_{k=1}^{m-1} \Omega\left(s_{k}\right)\right)=0, \quad j=0,1,2,3,4,
$$

where the nodes $s_{l+1}=s_{l}+h, l=0,1, \ldots, m, s_{0}=0$ and $h_{j}=\frac{t_{j}}{m}, \Omega(s)=$ $\left(s+t_{j}\right) e^{C^{T} P(s)}$.

Eq.(17) presents non-linear system of $N+1$ algebraic equations in the coefficients $c_{i}$. By solving it by using the Newton iteration method with suitable initial solution we obtain

$$
c_{0}=0.0002, \quad c_{1}=0.9895, \quad c_{2}=0.0022, \quad c_{3}=-0.0088, \quad c_{4}=0.0023 .
$$

Therefore, the approximate solution of this example can be found using (16) as follows

$$
x(t)=0.0002 P_{0}(t)+0.9895 P_{1}(t)+0.0022 P_{2}(t)-0.0088 P_{3}(t)+0.0023 P_{4}(t) .
$$

The exact solution of this problem is $x(t)=t$.

The behavior of the approximate solution using the proposed method with $N=4$ and the exact solution are presented in Fig.2. From this Fig., it is clear that the proposed method can be considered as an efficient method to solve the nonlinear integral equations. 


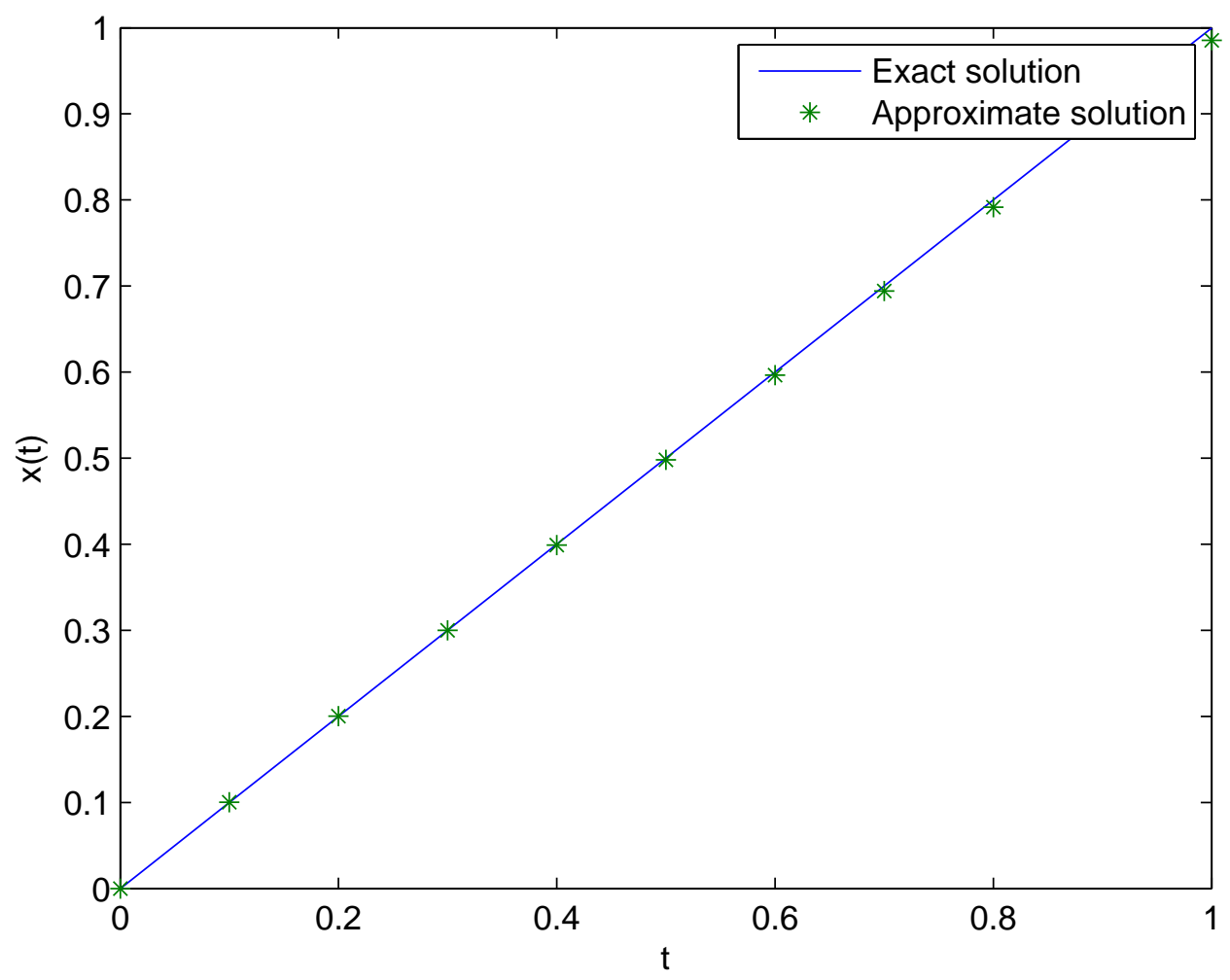

Fig.2. The behavior of the exact solution and the approximate solution at

$$
N=4 \text {. }
$$

\section{Example 3.}

Consider Eq.(1) with the following functions and coefficients

$$
\begin{gathered}
f(t)=t e+1, \quad \lambda_{1}=-1, \quad \lambda_{2}=1, \quad K_{1}(t, s)=s+t, \\
K_{2}(t, s)=0, \quad F(x(s))=e^{x(s)}, \quad G(x(s))=0 .
\end{gathered}
$$

Eq.(1) takes the following form

$$
x(t)=t e+1-\int_{0}^{1}(s+t) e^{x(s)} d s .
$$

The exact solution of this problem is $x(t)=t$.

We apply the suggested method with $N=3$, and approximate the solution $x(t)$ as in (16), and by the same procedure in the previous section with using Eq.(9) we have

$$
\sum_{i=0}^{3} c_{i} P_{i}\left(t_{j}\right)-f\left(t_{j}\right)+\frac{h}{2}\left(\Omega\left(s_{0}\right)+\Omega\left(s_{m}\right)+2 \sum_{k=1}^{m-1} \Omega\left(s_{k}\right)\right)=0, \quad j=0,1,2,3,
$$


where $s_{l+1}=s_{l}+h, l=0,1, \ldots, m, s_{0}=0$ and $h=\frac{1}{m}$ and $\Omega(s)=\left(s+t_{j}\right)$. $e^{\left(\sum_{i=0}^{3} c_{i} P_{i}(s)\right)}$.

Eq.(19) presents non-linear system of algebraic equations. By solving it using the well known Newton iteration method with suitable initial solution we obtain

$$
c_{0}=-0.0023, \quad c_{1}=1.0013, \quad c_{2}=0.0 \quad c_{3}=0.0 .
$$

Therefore, the approximate solution of this example can be found using (16)

$$
x(t)=-0.0023 P_{0}(t)+1.0013 P_{1}(t)+0.0 P_{2}(t)+0.0 P_{3}(t) .
$$

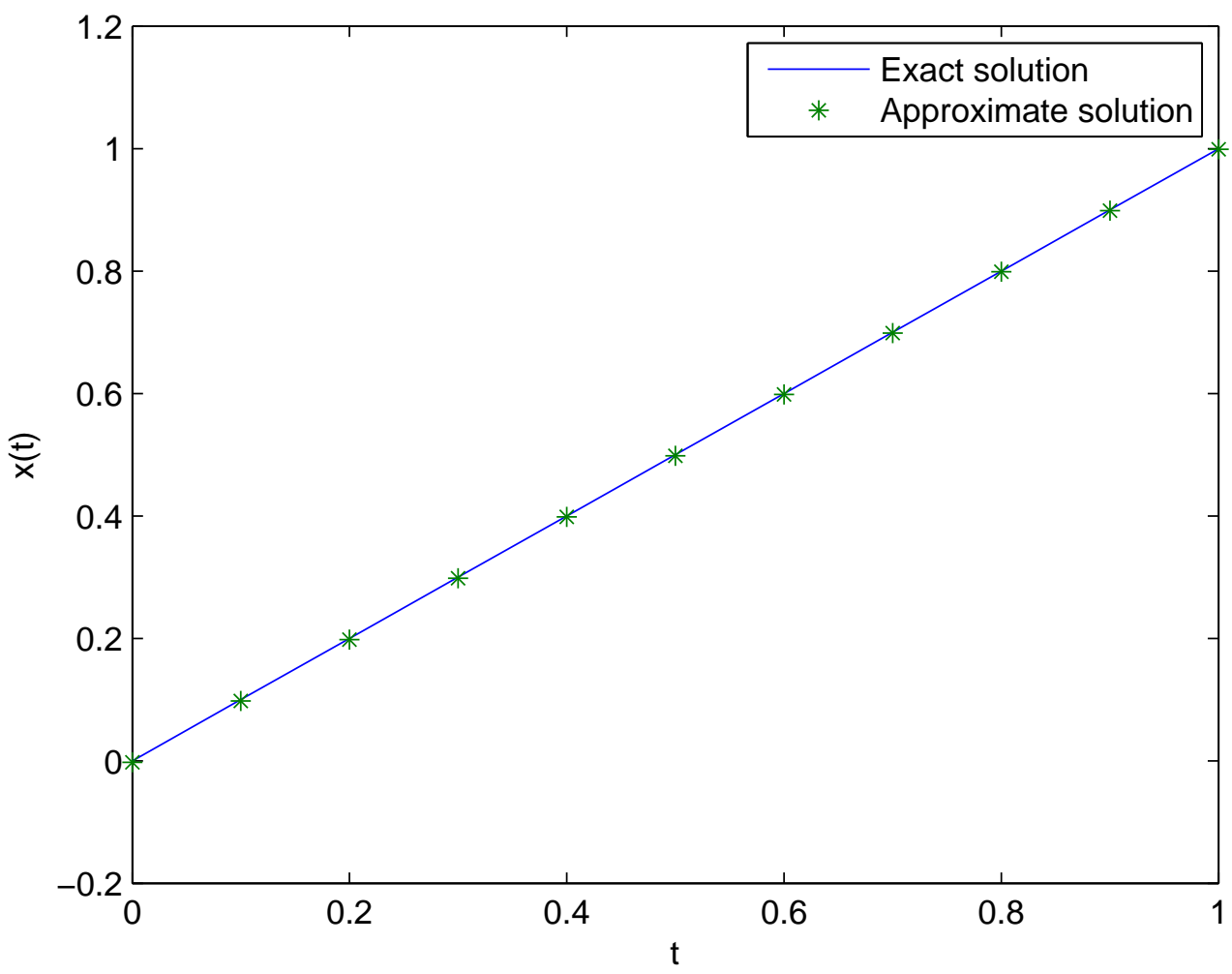

Fig.3. The behavior of the exact solution and the approximate solution at $N=3$.

The behavior of the approximate solution using the proposed method with $N=3$ and the exact solution are presented in Fig.3. 


\section{Example 4.}

Consider Eq.(1) with the following functions and coefficients

$$
\begin{gathered}
f(t)=\frac{t}{2}-\frac{t^{4}}{12}-\frac{1}{3}, \lambda_{1}=1, \quad \lambda_{2}=-1, \quad K_{1}(t, s)=s+t \\
K_{2}(t, s)=s-t, \quad F(x(s))=x(s), \quad G(x(s))=x^{2}(s) .
\end{gathered}
$$

Eq.(1) takes the following form

$$
x(t)=\frac{t}{2}-\frac{t^{4}}{12}-\frac{1}{3}+\int_{0}^{1}(s+t) x(s) d s+\int_{0}^{t}(s-t) x^{2}(s) d s .
$$

We apply the suggested method with $N=4$, and approximate the solution $x(t)$ as follows:

$$
x_{N}(t)=\sum_{i=0}^{4} c_{i} P_{i}(t)=C^{T} P(t) .
$$

By the same procedure in the previous section and using Eq.(9) we have

$$
\begin{aligned}
\sum_{i=0}^{4} c_{i} P_{i}\left(t_{j}\right)-f\left(t_{j}\right)-\frac{h}{2}\left(\Omega_{1}\left(\bar{s}_{0}\right)+\Omega_{1}\left(\bar{s}_{m}\right)\right. & \left.+2 \sum_{k=1}^{m-1} \Omega_{1}\left(\bar{s}_{k}\right)\right)-\frac{h_{j}}{2}\left(\Omega_{2}\left(s_{0}\right)+\Omega_{2}\left(s_{m}\right)\right. \\
& \left.+2 \sum_{k=1}^{m-1} \Omega_{2}\left(s_{k}\right)\right)=0,
\end{aligned}
$$

where $\bar{s}_{l+1}=\bar{s}_{l}+h, \quad s_{l+1}=s_{l}+h_{j}, l=0,1, \ldots, m, \quad s_{0}=\bar{s}_{0}=0, \quad h=\frac{1}{m}$, $h_{j}=\frac{t_{j}}{m}$, and $\Omega_{1}(s)=\left(s+t_{j}\right)\left(\sum_{i=0}^{4} c_{i} P_{i}(s)\right), \quad \Omega_{2}(s)=\left(t_{j}-s\right)\left(C^{T} P(s)\right)^{2}$. Eq.(22) presents non-linear system of $N+1$ algebraic equations. By solving it using Newton iteration method we obtain

$$
c_{0}=-0.0012, \quad c_{1}=0.9987, \quad c_{2}=-0.0039, \quad c_{3}=0.0007, \quad c_{4}=-0.0017 .
$$

Therefore, the approximate solution of this example can be found using (21):

$x(t)=-0.0012 P_{0}(t)+0.9987 P_{1}(t)-0.0039 P_{2}(t)+0.0007 P_{3}(t)-0.0017 P_{4}(t)$.

The exact solution of this problem is $x(t)=t$.

The behavior of the approximate solution using the proposed method using $N=4$ and the exact solution are presented in Fig.4. From Fig.4, it is clear that the proposed method can be considered as an efficient method to solve the nonlinear Hammerstein integral equations. 


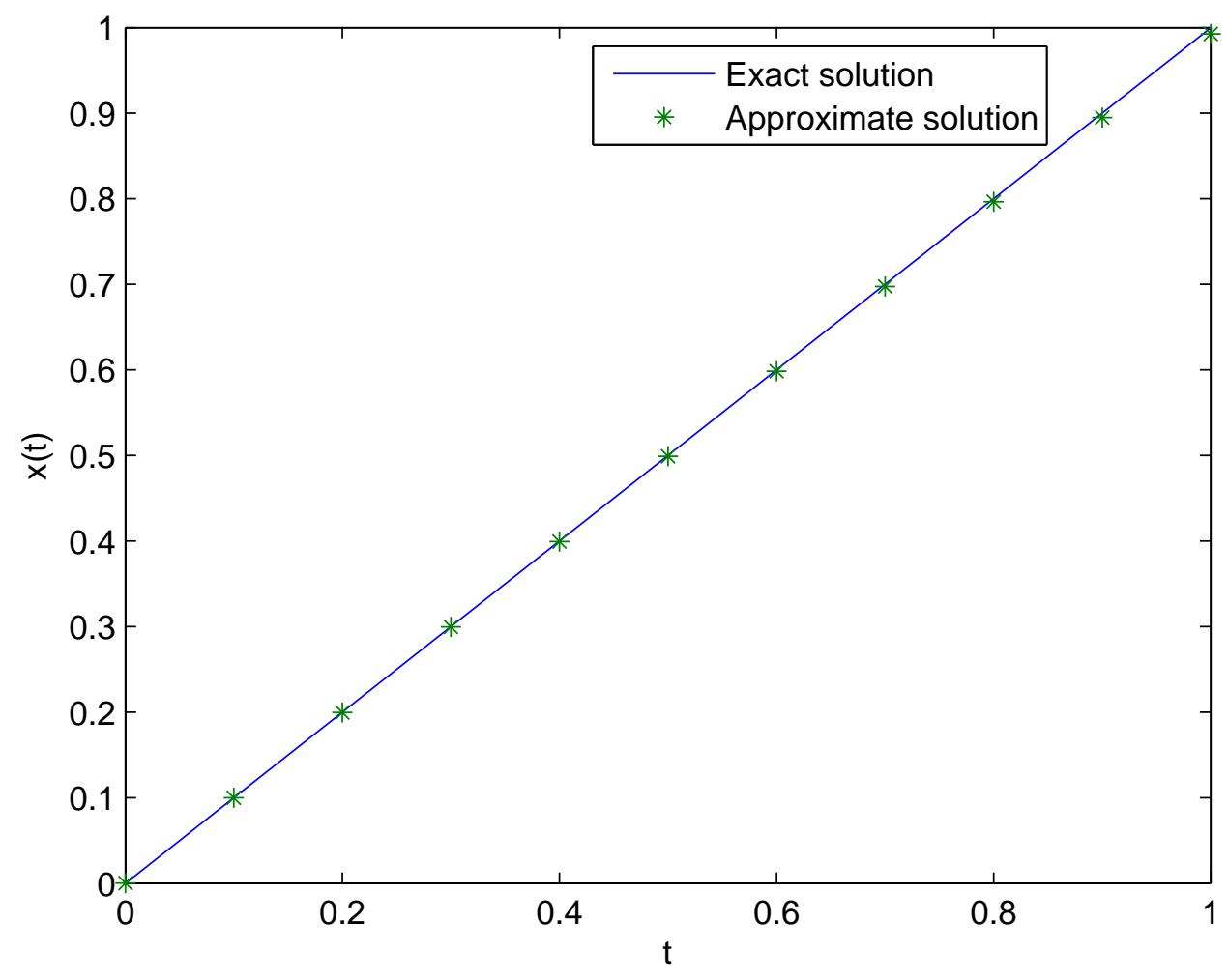

Fig.4. The behavior of the exact solution and the approximate solution at $N=4$.

\section{Conclusion}

An approximate method for the solution of linear and non-linear FredholmVolterra integral equations in the most general form has been proposed and investigated. For this purpose, the presented method which is based on the Legendre polynomials is proposed to find the approximate solution. A comparison of the exact solution reveals that the presented method is very effective and convenient. The numerical results show that the accuracy improves with increasing $N$, hence for better results, using number $N$ is recommended. Also, from the obtained approximate solution, we can conclude that the proposed method gives the solution in an excellent agreement with the exact solution. All computations are done using Matlab programming.

\section{ACKNOWLEDGEMENTS}


The authors are very grateful to the editor and referees for carefully reading the paper and for their comments and suggestions which have improved the paper.

\section{References}

[1] R.P. Agarwal, Boundary value problems for higher order integrodifferential equations, Nonlinear Anal. Theory Methods Appl., 9 (1983) 259-270.

[2] L.C. Andrews, Special Functions For Engineers and Applied Mathematicians, Macmillan publishing company, New York, (1985).

[3] E. Babolian, F. Fattahzadeh, E. Golpar Raboky, A Chebyshev approximation for solving nonlinear integral equations of Hammerstein type, Applied Mathematics and Computation, 189 (2007) 641-46.

[4] A.K. Borzabadi, A.V. Kamyad, H.H. Mehne, A different approach for solving the nonlinear Fredholm integral equations of the second kind, $A p$ plied Mathematics and Computation, 173 (2006) 724-735.

[5] L.M. Delves, J.L. Mohamad, Computational Methods for Integral Equations, Cambridge University press, (1985).

[6] M.M. Khader, A.S. Hendy, The approximate and exact solutions of the fractional-order delay differential equations using Legendre pseudospectral method, International Journal of Pure and Applied Mathematics, 74(3) (2012) 287-297.

[7] M.M. Khader, N.H. Sweilam, A.M.S. Mahdy, An efficient numerical method for solving the fractional diffusion equation, Journal of Applied Mathematics and Bioinformatics, 1 (2011) 1-12.

[8] M.M. Khader, Introducing an efficient modification of the homotopy perturbation method by using Chebyshev polynomials, Arab Journal of Mathematical Sciences, 18 (2012) 61-71.

[9] M.M. Khader, Numerical solution of nonlinear multi-order fractional differential equations by implementation of the operational matrix of fractional derivative, Studies in Nonlinear Sciences, 2(1) (2011) 5-12.

[10] S.T. Mohamed, M.M. Khader, Numerical solutions to the second order Fredholm integro-differential equations using the spline functions expansion, Global Journal of Pure and Applied Mathematics, 34 (2011) 21-29.] 
[11] N. Kurt, M. Sezer, Polynomial solution of high-order linear Fredholm integro-differential equations with constant coefficients, Journal of the Franklin Institute, 345 (2008) 839-850.

[12] M. Shahrezaee, Solving an integro-differential equation by Legendre polynomials and Block-pulse functions, Dynamical Systems and Applications, (2004) 642-647.

[13] N.H. Sweilam, Fourth order integro-differential equations using variational iteration method, Comput. Maths. Appl., 54 (2007) 1086-1091.

[14] N.H. Sweilam, M.M. Khader, R.F. Al-Bar, Homotopy perturbation method for linear and nonlinear system of fractional integro-differential equations, International Journal of Computational Mathematics and $\mathrm{Nu}$ merical Simulation, 1 (2008) 73-87.

[15] N.H. Sweilam, M.M. Khader, A Chebyshev pseudo-spectral method for solving fractional order integro-differential equations, ANZIAM, 51 (2010) $464-475$.

[16] N.H. Sweilam, M.M. Khader, Semi exact solutions for the bi-harmonic equation using homotopy analysis method, World Applied Sciences Journal, 13 (2011) 1-7.

[17] S. Yousefi, M. Razzaghi, Legendre wavelet method for the nonlinear Volterra-Fredholm integral equations, Math. Comp. Simul., 70 (2005) $1-8$. 